\title{
Towards precision medicine in bronchiectasis: what is the role of neutrophilic elastase determination?
}

\author{
Grace Oscullo Yepez $\mathbb{1}^{1}$, Jesus María Sierra-Párraga², Tomás Posadas \\ Blazquez ${ }^{1}$ and Miguel Ángel Martínez-García ${ }^{1}$
}

Affiliations: ${ }^{1}$ Pneumology Dept, Hospital Universitario y Politécnico La Fe, Valencia, Spain. ${ }^{2}$ Dept of Internal Medicine, University Medical Center Rotterdam, Rotterdam, The Netherlands.

Correspondence: Miguel Ángel Martínez-García, Pneumology Dept, Hospital Universitario y Politécnico La Fe, Bulevar Sur s/n, 46012-Valencia, Spain. E-mail: mianmartinezgarciadagmail.com

@ERSpublications

Biomarkers will have an important role in the diagnosis, prognosis and response to treatment in bronchiectasis during the transition towards precision medicine http://bit.ly/30swlUK

Cite this article as: Oscullo Yepez G, Sierra-Párraga JM, Posadas Blazquez T, et al. Towards precision medicine in bronchiectasis: what is the role of neutrophilic elastase determination? Eur Respir J 2019; 53: 1900765 [https://doi.org/10.1183/13993003.00765-2019].

The 21 st century will mark the transition from disease-centred medicine to patient-centred medicine. Medicine will evolve from the consideration of just a few symptoms and signs, and a limited number of complementary tests used to define most diseases, to the realisation that diseases are heterogeneous and dynamic. This change of paradigm will also mean that the current use of just a tiny fraction of the information provided by complementary tests will become outdated, and will result in an optimisation of most, if not all, of these findings. For example, less than $1 \%$ of the valid information provided by computed tomography of the chest, the gold standard for bronchiectasis diagnosis, is currently used. In short, this century will be the era of research into the complexity of diseases and the individualisation of treatment, based on the principles underlying what is known as "precision medicine". We may still seem a long way from achieving these objectives, but there is no doubt that the necessary tools have been developing in leaps and bounds. We now have the capacity to efficiently handle vast amounts of data (big data) and glean valid information from them via techniques based on artificial intelligence [1]. There have also been significant advances in "-omics" and the relationships between them (multi-omics) and system biology; this will surely lead to an improved genotypic, phenotypic and endotypic characterisation of disease and, therefore, a better identification of both specific therapeutic targets and the directions to be followed by future research [2]. All this makes the identification and measurement of different biological biomarkers a field of research with enormous potential.

Regarding airway diseases in general, and bronchiectasis in particular, it has proved extremely difficult to find useful biological biomarkers regarding diagnosis, prognosis or response to treatment [3]. This difficulty can probably be explained by the substantial heterogeneity and complexity in both the genotypic and phenotypic aspects of respiratory diseases. Furthermore, the discovery of a suitable biomarker is not an end in itself, as it then needs to be integrated into clinical practice as effectively as possible. In other words, its use must become generalised in various settings, geographical areas or clinically relevant subgroups of patients. Moreover, the techniques used should be easily performed, rapidly accessible and financially viable [4]. 
Neutrophilic elastase (NE) is a $29-\mathrm{kDa}$ serine protease released from the azurophilic granules of neutrophils, and is directly involved in the pathogenesis of bronchiectasis. It degrades elastin present in the airways and impairs the immune response of the patient against pathogenic microorganisms, making it one of the biological biomarkers that has been most widely discussed and studied $[5,6]$.

In this issue of the European Respiratory Journal, SHOEmark et al. [7] have contributed scientific evidence on the role of NE in bronchiectasis, following the group's classic line of research. However, the main focus of this study was not the known relationship between the concentration of NE and prognosis and severity of bronchiectasis $[8,9]$, but the validation of a new technique for measuring the concentration of $\mathrm{NE}$ in sputum: NEATstik. This point-of-care test does not require full processing of the sputum and provides rapid (within minutes) semi-quantitative measurement (scoring samples from 0 to 10 points) of $\mathrm{NE}$ concentration. This enables its use in everyday clinical practice, in both health centres and the home, and even as an end-point for clinical trials in bronchiectasis [7]. The authors validated this method in one series of 124 patients with stable bronchiectasis from two European centres and another of 50 patients from a third European centre that served as an external validation cohort. The measurement of NE by this method closely correlates with that obtained with the classical method of immunoassay, as well as presenting a similarly high correlation between independent observers and an adequate external validation. Furthermore, NE was able to predict future exacerbations and correlated with various parameters of severity, clinical data and quality of life in bronchiectasis patients. It is also worth noting that this method showed a high specificity for bronchial infection by Pseudomonas aeruginosa, which could prove very useful for ruling out this infection in the event of low concentration of NE, without any need for sputum cultures [7].

In spite of the limitations of the study, as acknowledged by the research group, particularly the preponderance of patients with severe bronchiectasis and the lack of external validation beyond the European context, studies such as this are very welcome, as they enable us to gradually understand the complexity of the disease and introduce new parameters for its evaluation, such as biomarkers, into clinical practice.

The advantages of measuring NE in bronchiectasis patients include: the numerous new methods for its assessment, one of which is already validated [7, 10]; its relationship with disease severity and prognosis $[8,9]$, as well as, to a lesser extent, response to conventional treatment of bronchiectasis [8]; and the possibility of developing specific drugs targeted toward this biomarker [11, 12], which has been attempted on several occasions, albeit with little success so far $[13,14]$. Moreover, there have been improvements in our understanding of the genetic information controlling the genesis of NE [15]. The main disadvantage of using NE as a biomarker in bronchiectasis, and thus the challenge that it faces in the near future, is its lack of specificity, since it is also present in high concentrations in chronic obstructive pulmonary disease, cystic fibrosis and, more generally, processes characterised by neutrophilic inflammation [5, 6]. Further disadvantages are the existence of other molecules with similar functions (i.e. serine proteases such as cathepsin G and proteinase 3) that could simulate the proteolytic capacity of NE even when the NE concentration is not particularly high [16]. In addition, the high levels of activity shown by the antiproteases (especially alpha-1 antitrypsin, a molecule that has been very well characterised both genotypically and endotypically), could reduce the effect of NE even in high concentrations [5]. It could be that the latter issue can be overcome by determining and analysing the specific relevance of various fundamental molecules involved in the balance of proteases/antiproteases in the lung, particularly in the formation and evolution of bronchiectasis, or by assessing the diagnostic and prognostic capacity and response to treatment of $\mathrm{NE}$ in conjunction with other biomarkers of interest in clinical practice (number of eosinophils or neutrophils, other markers of neutrophilic inflammation or bronchial infection, etc.). Furthermore, although the concentration of NE was predictive of exacerbations, it did not rise significantly when these occurred [7], suggesting that it is not an ideal marker for diagnosing exacerbations, probably due to the considerable endotypic heterogeneity in bronchiectasis. Surprisingly, $17 \%$ of patients with bronchiectasis presented normal NE values in the study reported by SHOEmark et al. [7]. This is a very interesting finding as it raises the possibility of describing a new phenotype of bronchiectasis that could have a different underlying endotype (inflammation) and could benefit further from personalised treatment approaches. Whatever the case, it seems clear that NE is a key molecule in the pathophysiology of bronchiectasis in most patients, and that its application as a biomarker in clinical practice would be enhanced if it were to be determined more rapidly.

Precision medicine for bronchiectasis is not a mirage. Some steps have already been taken in the right direction and clinical phenotypes that can be differentiated from the clinical, prognostic and response-to-treatment viewpoints are gradually emerging $[17,18]$. These findings point towards a more individualised approach, even though the pathophysiological knowledge (endotypes) on which these phenotypes may be based is still sparse [2]. Oncology is a speciality that is obtaining excellent results in 
the determination of new biomarkers for prognostic and therapeutic use; it provides us with a good point of reference and suggests that we are maybe closer to achieving our objectives than we think. Biomarkers undoubtedly play a key part in this process and it is possible that NE will be very useful once we know more about its role in bronchiectasis and, more particularly, its suitability for diagnosis, prognosis and assessment of treatment response.

Conflict of interest: None declared.

\section{References}

1 Miraza B, Wang W, Wang J, et al. Machine learning and integrative analysis of biomedical big data. Genes (Basel) 2019; 10: E87.

2 Flume PA, Chalmers JD, Olivier KN. Advances in bronchiectasis: endotyping, genetics, microbiome, and disease heterogeneity. Lancet 2018; 392: 880-890.

3 Wheelock CE, Goss VM, Balgoma B, et al. Application of 'omics technologies to biomarker discovery in inflammatory lung diseases. Eur Respir J 2013; 42: 801-825.

4 Hollander Z, DeMarco ML, Sadatsafavi M, et al. Biomarker development in COPD: Moving from P values to products to impact patient care. Chest 2017; 151: 455-467.

5 Polverino E, Rosales-Mayor E, Dale GE, et al. The role of neutrophil elastase inhibitors in lung diseases. Chest 2017; 152: 249-262.

6 Gramegna A, Amati F, Terranova L, et al. Neutrophil elastase in bronchiectasis. Respir Res 2017; 18: 211.

7 Shoemark A, Cant E, Carreto L, et al. A point-of-care neutrophil elastase activity assay identifies bronchiectasis severity, airway infection and risk of exacerbation. Eur Respir J 2019; 53: 1900303.

8 Chalmers JD, Moffitt KL, Sanchez-Cuartin G, et al. Neutrophil elastase activity is associated with exacerbations and lung function decline in bronchiectasis. Am J Respir Crit Care Med 2017; 195: 1384-1393.

9 Keir HR, Fong CJ, Crichton ML, et al. Personalised anti-inflammatory therapy for bronchiectasis and cystic fibrosis: selecting patients for controlled trials of neutrophil elastase inhibition. ERJ Open Res 2019; 5: 00252-2018.

10 Keir HR, Fong CJ, Dicker AJ, et al. Profile of the ProAxsis active neutrophil elastase immunoassay for precision medicine in chronic respiratory disease. Expert Rev Mol Diagn 2017; 17: 875-884.

11 Palmér R, Mäenpää J, Jauhiainen A, et al. Dipeptidyl peptidase 1 inhibitor AZD7986 induces a sustained, exposure-dependent in neutrophil elastase activity in healthy subjects. Clin Pharmacol Ther 2018; 104: 1155-1164.

12 von Nussbaum F, Li VM, Allerheiligen S, et al. Freezing the bioactive conformation to boost potency: the identification of BAY 85-8501, a selective and potent inhibitor of human neutrophil elastase for pulmonary diseases. Chem Med Chem 2015; 10: 1163-1173.

13 Stockley R, De Soyza A, Gunawardena K, et al. Phase II study of a neutrophil elastase inhibitor (AZD9668) in patients with bronchiectasis. Respir Med 2013; 107: 524-533.

14 Watz H, Nagelschmitz J, Kirsten A, et al. Safety and efficacy of the human neutrophil elastase inhibitor BAY 858501 for the treatment of non-cystic fibrosis bronchiectasis: a randomized controlled trial. Pulm Pharmacol Ther 2019; 56: 86-93.

15 Nikolic A, Milosevic K, Boskovic S, et al. Neutrophil elastase gene polymorphisms: modulators of response to therapy in childhood bronchiectasis? Lung 2014; 192: 595-599.

16 Korkmaz B, Moreau T, Gauthier F. Neutrophil elastase, proteinase 3 and cathepsin G: physicochemical properties, activity and physiopathological functions. Biochimie 2008; 90: 227-242.

17 Aliberti S, Lonni S, Dore S, et al. Clinical phenotypes in adult patients with bronchiectasis. Eur Respir J 2016; 47: $1113-1122$.

18 Martinez-Garcia MA, Vendrell M, Girón R, et al. The multiple faces of non-cystic fibrosis bronchiectasis. A Cluster analysis approach. Ann Am Thorac Soc 2016; 13: 1468-1475. 